OPEN ACCESS

Edited by:

John J. Foxe,

Albert Einstein College of Medicine,

USA

Reviewed by:

Ted S. Altschuler,

Albert Einstein College of Medicine,

USA

Brian P. Keane,

Rutgers University Center for

Cognitive Science, USA

Marina Shpaner.

UVM School

of Medicine, USA

*Correspondence:

Emma Gowen,

Faculty of Life Sciences,

University of Manchester, Carys

Bannister building, Dover Street,

Manchester, M13 9PL, UK

emma.gowen@

manchester.ac.uk

Received: 12 December 2014

Accepted: 16 February 2015

Published: 10 March 2015

Citation:

Jachim S, Warren PA, McLoughlin N and Gowen E (2015) Collinear

facilitation and contour integration in autism: evidence for atypical visual integration.

Front. Hum. Neurosci. 9:115. doi: 10.3389/fnhum.2015.00115

\section{Collinear facilitation and contour integration in autism: evidence for atypical visual integration}

\author{
Stephen Jachim ${ }^{1}$, Paul A. Warren ${ }^{2}$, Niall McLoughlin ${ }^{1}$ and Emma Gowen ${ }^{1 *}$ \\ ${ }^{1}$ Faculty of Life Sciences, University of Manchester, Manchester, UK, ${ }^{2}$ Psychological Sciences, University of Manchester, \\ Manchester, UK
}

Autism spectrum disorder (ASD) is a neurodevelopmental disorder characterized by impaired social interaction, atypical communication and a restricted repertoire of interests and activities. Altered sensory and perceptual experiences are also common, and a notable perceptual difference between individuals with ASD and controls is their superior performance in visual tasks where it may be beneficial to ignore global context. This superiority may be the result of atypical integrative processing. To explore this claim we investigated visual integration in adults with ASD (diagnosed with Asperger's Syndrome) using two psychophysical tasks thought to rely on integrative processing-collinear facilitation and contour integration. We measured collinear facilitation at different flanker orientation offsets and contour integration for both open and closed contours. Our results indicate that compared to matched controls, ASD participants show (i) reduced collinear facilitation, despite equivalent performance without flankers; and (ii) less benefit from closed contours in contour integration. These results indicate weaker visuospatial integration in adults with ASD and suggest that further studies using these types of paradigms would provide knowledge on how contextual processing is altered in ASD.

\footnotetext{
Keywords: autism, Asperger's Syndrome, collinear facilitation, contour integration, enhanced perceptual functioning, weak central coherence
}

\section{Introduction}

Autism spectrum disorder (ASD) is a neurodevelopmental disorder characterized by deficits in social communication and social interaction as well as repetitive and restricted behavior. Although diagnosed primarily by social symptoms (Lord et al., 2000), autism is often accompanied by unusual sensory experiences that can affect individual or multiple modalities. These can include hypersensitivity such as an aversion to bright or flashing lights or hyposensitivity, where the individual seems to be unaware or slow to respond to a stimulus that should normally elicit a response (Bogdashina, 2003). The importance of sensory symptoms is highlighted by their recent inclusion in the Diagnostic and Statistical Manual of Mental Disorders (American Psychiatric Association, 2013). Alongside these sensory symptoms, instances of perceptual superiority have also been reported, such as superior performance in visual search tasks where it may be beneficial to ignore global context (for reviews see Dakin and Frith, 2005; Simmons et al., 2009). Typically, in such tasks participants locate a particular stimulus or shape hidden among other "distractor" stimuli that form part of a larger, global scene while behavioral measures such as reaction times or error rates are taken to indicate performance. For example, ASD participants are often faster than neuro-typicals (NTs) at finding simple 
shapes embedded in more complex figures (Shah and Frith, 1983). This may be the result of perceiving an object in a disintegrated way-seeing it in terms of its "parts" rather than as a "whole" leading to the possibility that the perceptual advantage observed in ASD participants result from altered visual integration (Happé, 1996). Visual integration is a broad concept, but the current work focusses on the ability to integrate local information from different parts of a visual image into a larger, global percept such as a contour or shape. At the neurophysiological level, there is a large body of evidence that visual integration is mediated by interactions that occur between visual neurons, involving horizontal, feedforward and feedback connections (Kapadia et al., 2000; Angelucci et al., 2002; Nurminen and Angelucci, 2014). Visual neurons in early visual cortex (V1) are not only influenced by direct feedforward input from the environment, but also by horizontal (lateral) connections from neighboring cells and feedback from higher cortical areas (Shushruth et al., 2013). As this "contextual modulation" contributes to the detection, extraction and separation of shapes and objects from the background (Gheorghiu et al., 2014; Nurminen and Angelucci, 2014; Schmid and Victor, 2014) it is particularly relevant for understanding why perception is atypical in autism. Here we explore visual integration in ASD using two well-established psychophysical tasks that rely on contextual modulation: collinear facilitation and contour integration (for an in depth review of these tasks in relation to shape processing see Loffler (2008)).

In their seminal demonstration of collinear facilitation, Polat and Sagi (1993) showed that a faint Gabor target (a sinusoidal grating modulated by a Gaussian window) was easier to detect when flanked by high contrast, co-aligned Gabors compared to a no flanker (baseline) condition (Figure 1A). This phenomenon is critically dependent on several parameters including the orientation offset of the flankers relative to the target Gabor and the distance between the flankers and the target. Collinear facilitation is strongest when the target and flankers are coaligned $\left(0^{\circ}\right.$ offset, Figure 1A) compared to when the orientation of the flankers and target are offset (Figure 1B). In addition, collinear facilitation is strongest when the target and flankers are separated by approximately $3 \lambda(1 \lambda=1$ wavelength, the combined width of a single light and dark Gabor stripe), decreasing at larger distances, with suppression occurring for distances $<2 \lambda$. Collinear facilitation is thought to be mediated by propagation of excitatory signals from flanker to target cells along intrinsic horizontal (lateral) connections in V1 (Rockland and Lund, 1982; Gilbert and Wiesel, 1983; Livingstone and Hubel, 1984; Gilbert and Wiesel, 1989; Polat and Sagi, 1993). These connections are more numerous between cells of similar orientation preference, which could account for greater collinear facilitation found when the target and flankers are aligned (Kapadia et al., 1995; Fitzpatrick, 1996; Stettler et al., 2002). However, when target and flanker stimuli are positioned $<2 \lambda$ apart, suppression occurs via the horizontal connections, termed lateral inhibition (Hartline, 1949; Blakemore et al., 1970; Polat and Sagi, 1993). There is also likely to be feedback from higher cortical areas influencing contextual effects in V1 (Hupé et al., 1998; Angelucci et al., 2002; Freeman et al., 2003; Huang and Hess, 2008).
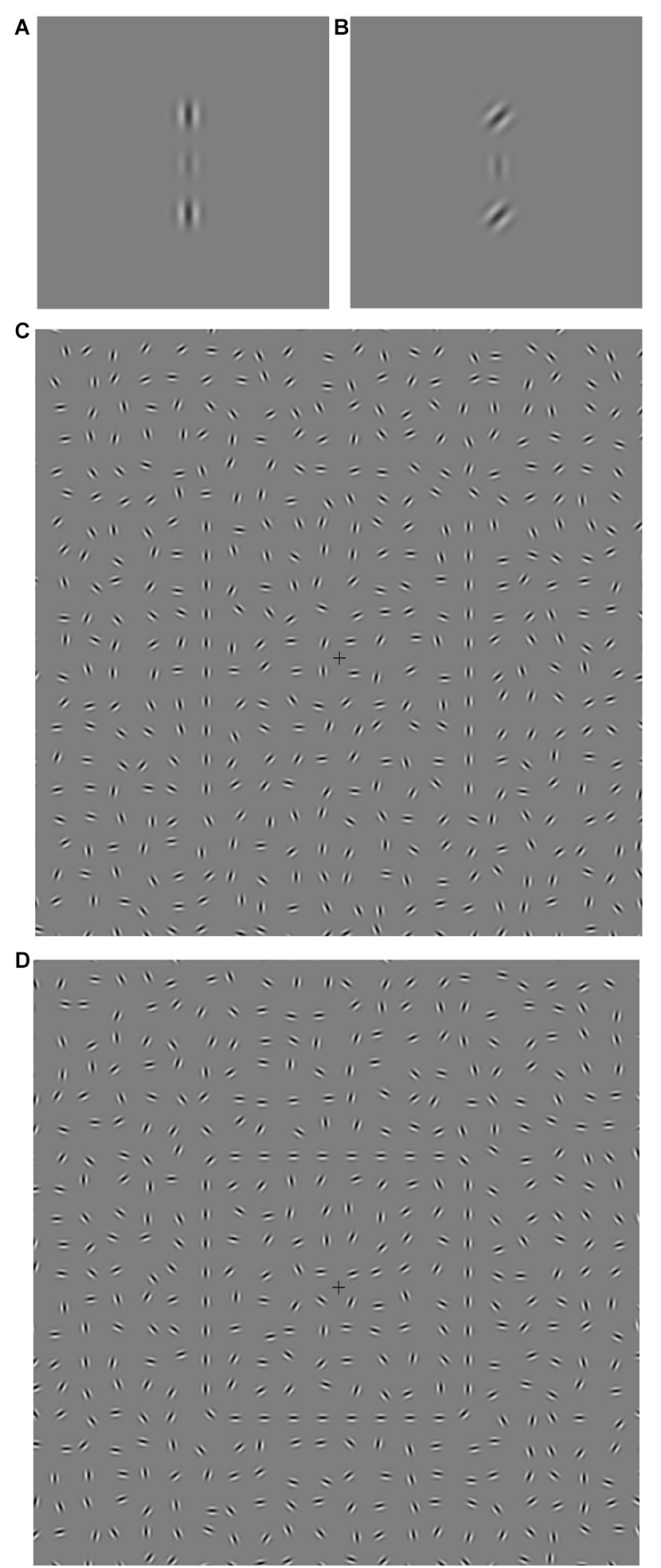

FIGURE 1 | (A-D) Central area screenshots of the stimuli used for Experiment 1 (collinear facilitation, A,B) and Experiment 2 (contour integration, C,D). (A) Flankers are in the vertically aligned condition ( $0^{\circ}$ orientation offset). The separation between the central target and each vertically aligned flanker is $3 \lambda$ (one $\lambda$ is equal to one Gabor cycle or wavelength, i.e., the combined width of a single light and dark stripe). (B) An example of one of the three orientation (Continued) 


\section{FIGURE 1 | Continued}

offset conditions, in this case the flanker offset is $45^{\circ}$. (C) Open contour: lines made up of aligned Gabor elements are embedded in a background of randomly oriented Gabors. (D) Closed contour: a square made up of aligned Gabor elements is embedded in a background of randomly oriented Gabors.

Feedback projections may enhance collinear contour elements by excitation (Shmuel et al., 2005), or by attentional modulation (Freeman et al., 2004).

Only one previous study has investigated collinear facilitation in autism (Kéita et al., 2011). They found more facilitation for an autistic group than a control group when collinear flankers were separated from the target Gabor by $3 \lambda$. The authors argued that these findings support altered lateral connectivity in autism, which in turn could lead to atypical responses to contextual information and be partly responsible for enhanced perceptual functioning in ASD. In order to extend these findings, our first aim was to examine collinear facilitation in adults with autism but using a different flanker condition: Instead of distance, we used four flanker orientation offsets. Flanker orientation was chosen as it is a well-tested and reliable paradigm in the neuroptypical literature and can provide a detailed picture of the pattern of lateral connectivity as the strength of collinear facilitation is likely to result from different levels of connectivity between cells of different orientation preference (Kapadia et al., 1995; Fitzpatrick, 1996; Stettler et al., 2002). In line with the findings of Kéita et al. (2011), we predicted altered visual integration, demonstrated by higher collinear facilitation at the four flanker orientation offsets compared to the control group.

Our second aim was to examine visual integration using a Gabor based contour integration task. Contour integration involves the detection of Gabor elements that form a contour amidst a background of randomly oriented Gabors (Field et al., 1993; Figure 1C). Target detection is impaired when the relative orientation (jitter) of adjacent path elements is increased. Contours can either be open (e.g., a single line) or closed (e.g., a shape). Interestingly, Kovács and Julesz (1993) found that closed contours were easier to detect than non-closed contours, an effect they ascribe to the global stimulus structure of the closed contour. Later evidence further supports the idea of a separate global closure driven mechanism that is sensitive to the detection of closed contours (Mathes and Fahle, 2007; Gerhardstein et al., 2012). Like collinear facilitation, contour integration is thought to involve excitatory horizontal connections between cells of similar orientation preference within V1 (Rockland and Lund, 1982; Gilbert and Wiesel, 1983, 1989; Livingstone and Hubel, 1984; Field et al., 1993; Kovács and Julesz, 1993; Pettet et al., 1998; Tversky et al., 2004), and/or feedback from extrastriate areas (Hupé et al., 1998; Angelucci et al., 2002; Achtman et al., 2003; Altmann et al., 2003; Shpaner et al., 2013). Such feedback is likely to originate from areas responsible for simple shape processing such as the lateral occipital complex (LOC; Mijović et al., 2014) and may contribute to enhanced detection of closed contours (Gerhardstein et al., 2012). For example, when human observers viewed line drawings that varied from open lines to $2 \mathrm{D}$ and $3 \mathrm{D}$ shapes, the progression from open to closed stimuli was positively correlated with fMRI brain activity in the LOC, and negatively correlated with activity in V1 (Murray et al., 2002).

Contour integration provides a useful test of visual integration as it involves combining a number of separate elements into a larger, global percept, contributing to the grouping and segmentation of objects in the environment (Loffler, 2008). A number of studies have previously investigated contour integration in individuals with ASD, and they report conflicting results. Blake et al. (2003) asked children to identify the quadrant in which a circular shape composed of "line" stimuli was located and found no difference in contour detection between the autistic and control group. However, these line stimuli have been criticized for containing low spatial frequencies enabling grouping to occur via a low spatial frequency mechanism, as opposed to true grouping mechanisms (Dakin and Frith, 2005). More recent studies overcome this confound by using bandpass Gabor stimuli. Del Viva et al. (2006) used a computerized display of random Gabor distractors to test how well participants could locate the quadrant in which a circular Gabor contour was placed. They found equivalent performance when comparing autistic children to age-matched controls. Kemner et al. (2007) used a card-based version of the contour integration task with closed contour stimuli, again finding similar performance between autistic children and control groups. One limitation of these studies is that they use long stimulus presentation times ( $>1 \mathrm{~s}$ ), which may have hidden any differences if the ASD group required more time to distinguish the shape (Van der Hallen et al., 2014). More recent work has identified group differences. In an electrophysiological study, Pei et al. (2009) recorded visually evoked potentials while children watched a Gabor display that alternated every $500 \mathrm{~ms}$ between circular contours and random patterns. They failed to detect what they considered to be the neural correlate of contour integration in low functioning children with autism, suggesting that reduced contour integration may be present for shorter stimulus durations. Evers et al. (2014) reported that children with ASD were slower and less accurate than controls at identifying contours based on everyday objects, which gradually emerged from a background of randomly oriented Gabors.

These latter studies suggest that atypical contour integration in ASD may exist, but is only apparent when using more challenging protocols such as shorter stimulus durations, or object identification as opposed to detection of simple shapes. At present, it remains unclear whether alterations in contour integration can be found for more simple shape detection when using shorter presentation times. Furthermore, no study to date has compared open vs. closed shapes in individuals with ASD. This is potentially important given that the detection of closed contours may involve a separate global closure driven mechanism compared to open contours, and that integrating parts into a whole may be problematic for ASD (Happé and Frith, 2006; Van der Hallen et al., 2014). Finally, all previous studies examining contour integration in ASD have been performed on children, but we chose to study adults in order to use a more standardized, controlled psychophysical set up, as has been used previously with neurotypical adults (Field et al., 1993; Dakin and Baruch, 2009; Schumacher et al., 2011). Therefore, in the 
current study we assessed contour integration using relatively simple open and closed stimuli but with short presentation times. These two stimuli were presented with their Gabors arranged at different levels of orientation (jitter) ranging from 0 degrees (contour is easily detectable) to \pm 90 degrees (contour is difficult to detect). Contour integration ability was measured by determining the level of jitter at which a participant could successfully detect the target with $75 \%$ accuracy, termed jitter tolerance. If participants with ASD have impairments in visual integration, then we would expect weaker contour integration when compared to controls, particularly for closed contours.

\section{Materials and Methods}

\section{Participants}

Thirteen ASD participants (3 female) and thirteen healthy controls matched on age, sex, handedness and full scale IQ were recruited through local support groups and volunteer advertisements. This sample number was based on a pilot study examining collinear facilitation and contour integration in 17 neurotypical participants using G*power 6 (Faul et al., 2007). For the collinear facilitation experiment we calculated power for the main effect of orientation. There was an effect size of 1.13 and power of 0.99 , giving a sample size of 13 . For the contour integration experiment we calculated power for the difference between lines and squares. There was an effect size of 1.65 and power of 0.99 , giving a sample size of 9 . We were unable to do a power calculation for the autistic group, as this is the first time these particular paradigms have been used. However, a post hoc power test using our autistic participants indicates a power of 1 for the above comparisons.

ASD participants had been given a diagnosis by outside clinical assessment (World Health Organization, 1993; American Psychiatric Association, 1994) of Asperger's syndrome. Diagnosis was confirmed using module 4 of the Autism Diagnostic Observation Schedule by a qualified researcher (Lord et al., 2000). Demographics of the individual participants can be seen in Table 1. One participant fell just below the cutoff for ASD, likely due to his older age facilitating the development of compensatory strategies, which can make ADOS scoring more challenging. However, their data was included as statistical comparisons remained significant and in the same direction when this participant was removed. Age, Full Scale, Verbal and Performance IQ, measured using Wechsler Abbreviated Scale of Intelligence (Wechsler, 1999) did not differ significantly between the two groups (Table 1). Four of the participants in each group were left-handed, all had normal or corrected-to-normal vision (6/6), using reduced Snellens at $33 \mathrm{cms}$, with a lower limit of 6/6. All had $60^{\prime}$ stereo acuity measured using the TNO stereotest. Participants gave written informed consent and the study was approved by the University of Manchester Research Ethics Committee.

\section{Apparatus}

Stimuli for all experiments were displayed on an Iiyama MA203DT CRT monitor with a screen resolution of $1600 \times 1200$ and a vertical refresh rate of $85 \mathrm{~Hz}$. Participants were seated in a darkened room with the viewing distance to screen fixed at $70 \mathrm{~cm}$ by the use of a chinrest. The mean display luminance for all experiments was $42 \mathrm{~cd} / \mathrm{m}^{2}$. A video-switcher device gave true 12-bit luminance resolution ( $\mathrm{Li}$ et al., 2003). The video output was gamma corrected using a psychophysical procedure described by Li et al. (2003) and calibrated with a Minolta CS-100 Chroma meter colorimeter (Konica Minolta Sensing America Inc., Ramsey, New Jersey). Participant responses were collected via two color-coded keys on a standard UK computer keyboard.

\section{Stimuli}

For Experiment 1, stimuli consisted of target and flanker Gabors generated with Psychtoolbox (Brainard, 1997; Pelli, 1997; Figure 1). Each Gabor had a bandwidth of 1.14 octaves, standard deviation of $0.16^{\circ}$ and a spatial frequency of 3.0 cpd. The target Gabor was presented centrally and flanked vertically by two high contrast Gabors ( $60 \%$ Michelson contrast) with a separation of $3 \lambda$ ( $1^{\circ}$ of visual angle). The orientation offset of the flanking Gabor pair $\left(0^{\circ}, 15^{\circ}, 30^{\circ}\right.$ or $\left.45^{\circ}\right)$ gave four experimental conditions plus a baseline (no flankers) condition.

For Experiment 2, stimuli consisting of contour and background Gabors were generated and displayed by a Java program. Each Gabor had a bandwidth of 1.2 octaves, standard deviation of $0.1^{\circ}$ and a spatial frequency of $4.6 \mathrm{cpd}$. All Gabors were displayed at $100 \%$ Michelson contrast. The display was divided into a $33 \times 24$ invisible grid and each cell initially contained a randomly placed Gabor. The Gabor position was perturbed $\pm x$ pixels horizontally and $\pm y$ pixels vertically from the center of the cell, where $x$ and $y$ represent a pair of random integers independently sampled from a uniform distribution over the range $[0,11]$. Each Gabor was randomly oriented (jittered) within the range $\pm 90^{\circ}$. Experiment 2 used two different target stimuli; vertical lines (Figure 1C) and a square (Figure 1D). For the lines stimulus, the vertical columns of 10 Gabors $5^{\circ}$ to the left and right of the central fixation cross were replaced by columns of 10 Gabors that were centrally located within the cells to form a straight line. The jitter of the target Gabors varied from trial to trial. A comparison stimulus was created in the same way, but each of the Gabors in the two 10 Gabor columns were jittered within the range $\pm 90^{\circ}$ but still centrally located within the cells. This ensured that the only difference between the target presentation and the comparison presentation was the jitter of the Gabors that formed the target contour lines. The square stimulus (and square comparison) were formed in the same way, with the addition of two horizontal lines, and $\pm 45^{\circ}$ angled corner Gabors. The spatial location of the vertical lines was identical for both the lines and square stimuli and remained constant throughout the experiment. During each trial, all the Gabors forming the target contour were jittered by an amount from the uniformly distributed ranges $0^{\circ}$, $0-15^{\circ}, 0-30^{\circ}, 0-45^{\circ}, 0-60^{\circ}, 0-75^{\circ}$ or $0-90^{\circ}$ giving seven jitter conditions.

\section{Procedure}

All experiments used a two interval forced choice (2IFC) procedure and stimuli were viewed binocularly. The trial 
TABLE 1 | Participant demographics

\begin{tabular}{|c|c|c|c|c|c|c|c|c|c|c|c|c|c|c|}
\hline ASD & Age & Sex & Hand & FSIQ & PIQ & VIQ & ADOS & Controls & Age & Sex & Hand & FSIQ & PIQ & VIQ \\
\hline 1 & 25 & $\mathrm{~F}$ & $\mathrm{R}$ & 128 & 108 & 142 & 7 & 1 & 38 & $M$ & $\mathrm{R}$ & 129 & 128 & 132 \\
\hline 2 & 18 & $M$ & $\mathrm{~L}$ & 117 & 119 & 110 & 7 & 2 & 20 & $M$ & $\mathrm{R}$ & 127 & 111 & 136 \\
\hline 3 & 40 & $M$ & $\mathrm{R}$ & 134 & 121 & 138 & 10 & 3 & 34 & $\mathrm{~F}$ & $\mathrm{R}$ & 136 & 127 & 136 \\
\hline 4 & 30 & $\mathrm{~F}$ & $R$ & 131 & 119 & 136 & 8 & 4 & 19 & $M$ & $\mathrm{~L}$ & 133 & 123 & 136 \\
\hline 5 & 42 & $M$ & $\mathrm{~L}$ & 123 & 120 & 119 & 5 & 5 & 29 & $\mathrm{~F}$ & $\mathrm{R}$ & 124 & 121 & 120 \\
\hline 6 & 38 & $M$ & $\mathrm{~L}$ & 134 & 132 & 128 & 10 & 6 & 36 & $\mathrm{M}$ & $\mathrm{R}$ & 124 & 132 & 110 \\
\hline 7 & 39 & $M$ & $R$ & 118 & 116 & 117 & 7 & 7 & 22 & $M$ & $\mathrm{R}$ & 114 & 128 & 102 \\
\hline 8 & 21 & $M$ & $\mathrm{R}$ & 109 & 100 & 116 & 8 & 8 & 40 & $M$ & $\mathrm{R}$ & 107 & 97 & 118 \\
\hline 9 & 39 & $M$ & $\mathrm{~L}$ & 101 & 99 & 102 & 10 & 9 & 20 & $\mathrm{~F}$ & $\mathrm{~L}$ & 104 & 106 & 101 \\
\hline 10 & 23 & $M$ & $\mathrm{R}$ & 132 & 128 & 130 & 10 & 10 & 20 & $M$ & $\mathrm{R}$ & 121 & 123 & 115 \\
\hline 11 & 22 & $M$ & $R$ & 118 & 111 & 119 & 9 & 11 & 26 & $M$ & $\mathrm{R}$ & 138 & 126 & 141 \\
\hline 12 & 22 & $M$ & $\mathrm{R}$ & 118 & 119 & 114 & 10 & 12 & 38 & $M$ & $\mathrm{~L}$ & 118 & 119 & 103 \\
\hline 13 & 20 & $\mathrm{~F}$ & $\mathrm{R}$ & 105 & 102 & 106 & 10 & 13 & 34 & $\mathrm{M}$ & $\mathrm{L}$ & 123 & 119 & 120 \\
\hline Mean & 29.15 & & & 120.62 & 114.92 & 121.31 & & Mean & 28.92 & & & 122.92 & 120.00 & 120.77 \\
\hline $\mathrm{SD}$ & 9.07 & & & 11.00 & 10.34 & 12.55 & & SD & 8.08 & & & 10.31 & 9.93 & 14.28 \\
\hline $\begin{array}{l}\text { Group } \\
\text { comparison }\end{array}$ & $\begin{array}{l}t=0.07 \\
p=0.95\end{array}$ & & & $\begin{array}{l}t=-0.55 \\
p=0.625\end{array}$ & $\begin{array}{l}t=-1.28 \\
p=0.665\end{array}$ & $\begin{array}{l}t=0.102 \\
p=0.581\end{array}$ & & & & & & & & \\
\hline
\end{tabular}

Statistical comparison between the two groups on age, Full Scale IQ (FSIQ), Performance IQ (PIQ) and Verbal IQ NIQ) can be seen in bottom row.

sequence for Experiment 1 is shown in Figure 2A. Each stimulus presentation had a duration of $105 \mathrm{~ms}$ and was accompanied by an audible beep. Either the first or second stimulus presentation contained the central target Gabor and the participant indicated which interval the target appeared in by pressing one of two keys on the keyboard. Each of the five conditions (four flanker orientation offsets and a no-flanker baseline condition) were randomly interleaved during the experiment, each condition occurring once every five trials. Each condition was presented 60 times, giving a total of 300 trials. Participant target contrast thresholds were determined by varying the target contrast using the psi-method (Kontsevich and Tyler, 1999) as implemented by the Palamedes Matlab toolbox (Kingdom and Prins, 2009).

The trial sequence for Experiment 2 is shown in Figure 2B. Participants were instructed to focus on the fixation cross (which was always visible) throughout the entire experiment. The two stimulus presentations were presented for $130 \mathrm{~ms}$ each, separated by a blank screen containing the fixation cross for $505 \mathrm{~ms}$. Either the first or second stimulus presentation contained the target (Figure 1C), and the participant indicated which interval the target appeared in by pressing one of two keys on the keyboard. There were forty randomly ordered presentations of each of the seven jitter ranges, presented in four blocks of 70 trials. The participant's ability to tolerate jitter was determined using the method of constant stimuli (Field et al., 1993; Schumacher et al., 2011). After a short break the procedure was repeated using the square stimulus (Figure 1D). The order of the two stimuli was counter-balanced across participants.

As the contour integration experiment uses peripheral stimuli that might encourage saccades away from the central fixation cross, the left eye of each participant was tracked using GazeTracker software (San Agustin et al., 2010) and a remotely located camera. Nine point calibration with GazeTracker gave a spatial resolution of $0.25^{\circ}$, and eye movements were recorded and displayed on the experimenter's monitor using a custom Java
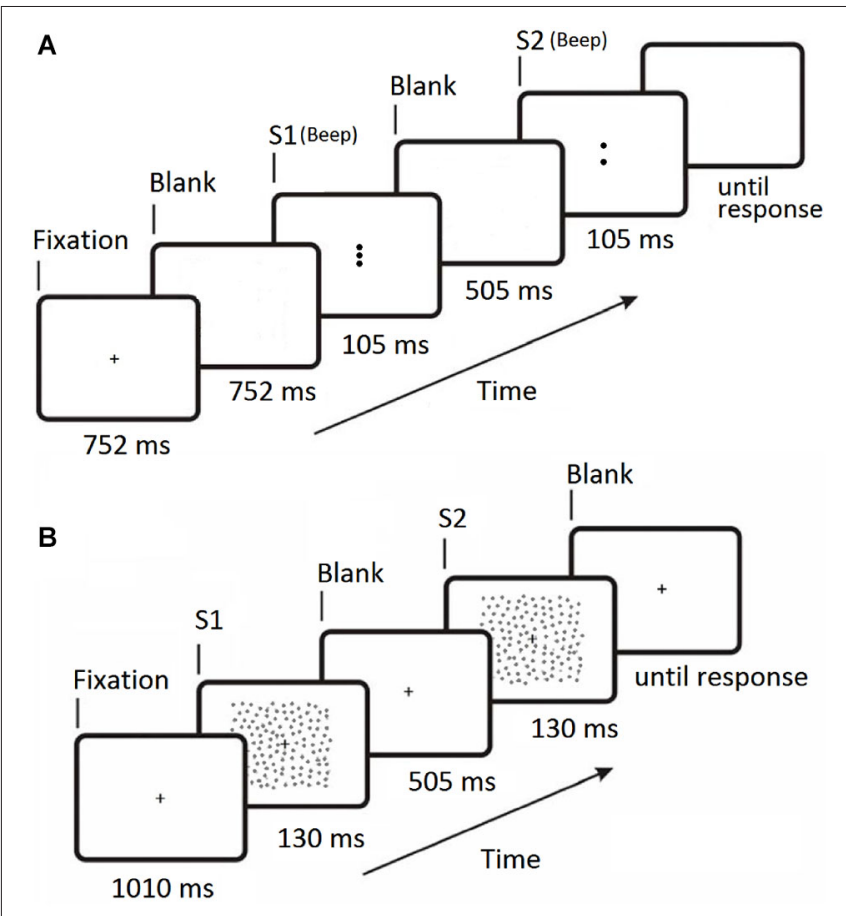

FIGURE 2 | Timelines for the two 2IFC trial sequences. (A) Experiment 1 (collinear facilitation). S1 is the first stimulus presentation period, S2 is the second stimulus presentation period. The central Gabor target appears randomly in either S1 or S2 on each trial and a $100 \mathrm{~ms}$ beep was sounded at the start of both S1 and S2. (B) Experiment 2 (contour integration). S1 is the first stimulus presentation period, $\mathrm{S} 2$ is the second stimulus presentation period. The contour target appears randomly in either S1 or S2 on each trial. For Experiment 2 the fixation cross remains on screen at all times.

application. Participants were informed by the experimenter if their gaze-point deviated from the fixation cross by more than $1.5^{\circ}$ in any direction. Eye-tracking co-ordinates for $84.3 \%$ of the trials were successfully recorded. For the ASD group, fixation 
for $86.4 \%$ of trials was within $\pm 1.5^{\circ}$ of cross. For the NT group, fixation for $94.3 \%$ of trials was within $\pm 1.5^{\circ}$ of the cross. Analysis performed after dropping trials recorded outside the fixation area did not significantly affect the results. We therefore used data from all trials in the reported analysis.

\section{Analysis}

For Experiment 1, the psi-method returned the running estimates of the contrast thresholds (Weibull distribution; $81.6 \%$ correct performance level) based on the posterior distribution for each of the five flanker conditions. Facilitation is measured in decibel units $(\mathrm{dB})$, which are $-20 \times \log _{10}$ value of the contrast ratio, the contrast ratios being defined as the threshold at each flanker orientation offset divided by the baseline threshold (Polat and Sagi, 1994). For Experiment 2 which used the method of constant stimuli, we fitted a logistic psychometric function using the Psignifit Matlab toolbox (version 2.5.6), ${ }^{1}$ which is based on Wichmann and Hill (2001). Performance over the seven jitter levels was determined using maximum-likelihood estimation. Thresholds were estimated at the $75 \%$ correct performance level. Correlations were performed between the two contour integration tasks and between the contour integration and collinear facilitation tasks.

\section{Experiment 3}

In a third experiment, we tested whether the improvement predicted with the square compared to lines stimulus was caused by a closed contour (Kovács and Julesz, 1993), rather than simply an increase in the number of GPs in the target (and therefore a more powerful stimulus). We repeated Experiment 2 with an additional stimulus (Figure 3 ). This "windmill" stimulus tested for an open contour condition having the same number of GPs as the square. We predicted that the windmill will be easier to detect than the lines, but harder to detect than the square. Stimuli were tested on a new NT group $(n=15$ (8 female), mean age $( \pm S D)$ $20.7 \pm 2.3$ ). All had normal or corrected-to-normal vision using reduced Snellens, and $60 \mathrm{~s}$ arc stereo acuity measured using the TNO stereotest.

\section{Results}

\section{Collinear Facilitation}

We investigated collinear facilitation for the ASD and NT groups by measuring facilitation at four different flanker orientation offsets. The NT $(M=0.035, S D=0.008)$ and ASD $(M=0.036$, $S D=0.007)$ baseline contrast thresholds were not significantly different $\left(t_{(24)}=0.28, p=0.78\right)$, indicating similar contrast sensitivity between the two groups (Figure 4A). Figure $4 \mathbf{B}$ shows the mean facilitation across the groups at different flanker orientation offsets. Consistent with previous studies, facilitation is highest when the flankers are vertically aligned with the target $\left(0^{\circ}\right.$ orientation offset) and decreases with increasing flanker orientation offset. A mixed ANOVA of group (NT and ASD) by flanker orientation offset $\left(0^{\circ}, 15^{\circ}, 30^{\circ}\right.$ and $\left.45^{\circ}\right)$ revealed a significant main effect of flanker orientation offset

\footnotetext{
${ }^{1}$ see http://bootstrap-software.org/psignifit/
}

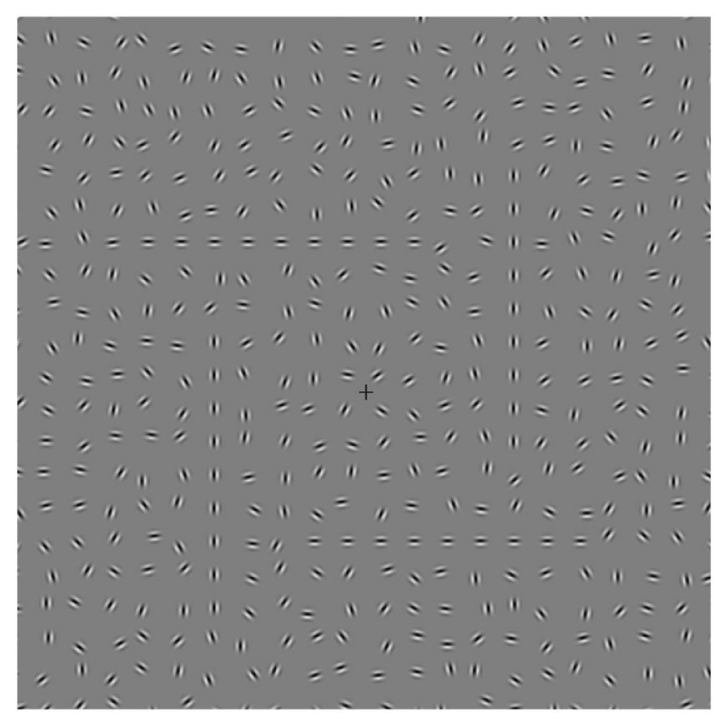

FIGURE 3 | Central area screenshot of experiment 3 showing the "windmill" contour embedded in a background of randomly oriented Gabors. This stimulus had the same number of Gabors as the square contour stimulus but with an "open" contour arrangement.

$\left(F_{(3,72)}=33.11, p<0.001, \eta_{P}^{2}=0.56\right)$ showing that overall, facilitation decreased as orientation offset increased $\left[0^{\circ}(M=\right.$ $4.41, S D=1.44), 15^{\circ}(M=3.34, S D=1.80), 30^{\circ}(M=2.57$, $\left.S D=1.62), 45^{\circ}(M=1.76, S D=1.48)\right]$. There was also a significant main effect of group $\left(F_{(1,24)}=5.65, p=0.021, \eta_{P}^{2}=\right.$ 0.20 ) indicating that the NT group showed more facilitation than the ASD group [NT $(M=3.56, S D=1.72)$, ASD $(M=2.41$, $S D=1.81)$ ]. Post-hoc paired $t$-tests (Bonferroni corrected) were performed, examining facilitation at each orientation between the two groups. They revealed a significant difference at an offset of $15^{\circ}$, [NT $(M=4.32, S D=1.60)$, ASD $(M=2.46, S D=$ $\left.1.61),\left(t_{(23)}=2.89, p=0.032\right)\right]$, indicating higher facilitation for NTs at this offset.The group $\times$ orientation offset interaction was not significant $\left(F_{(3,72)}=1.80, p=0.34, \eta_{P}^{2}=0.05\right.$. This result contradicts previous findings of higher facilitation in ASD (Kéita et al., 2011), but supports the idea of atypical integration in autism.

\section{Contour Integration}

We investigated contour integration by calculating jitter tolerance in degrees (the level of jitter at which a participant could successfully detect the target with $75 \%$ accuracy) for the ASD and NT groups in two stimulus conditions (lines and squares). Figure $\mathbf{5 A}$ shows the mean jitter tolerance across the groups for the different stimuli. A mixed ANOVA of group (NT and ASD) by stimulus (lines and square) revealed a significant main effect of stimulus $\left(F_{(1,24)}=46.26, p<\right.$ $\left.0.001, \eta_{P}^{2}=0.66\right)$ indicating that, In line with previous studies, detection was better for closed compared to open stimuli. There was no main effect of group $\left(F_{(1,24)}=0.98, p=0.33, \eta_{P}^{2}=\right.$ $0.04)$ but crucially, we found a significant interaction of group $\times$ stimulus $\left(F_{(1,24)}=7.00, p=0.014, \eta_{P}^{2}=0.23\right)$. Further investigation of the interaction showed that NT participants 


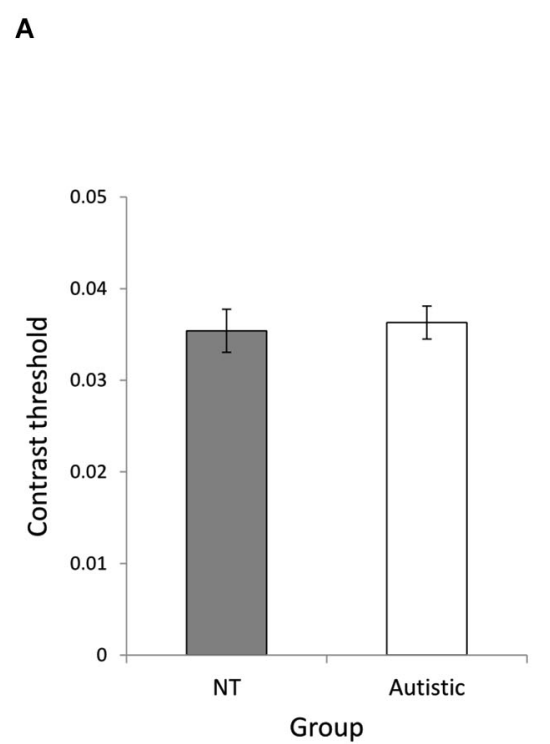

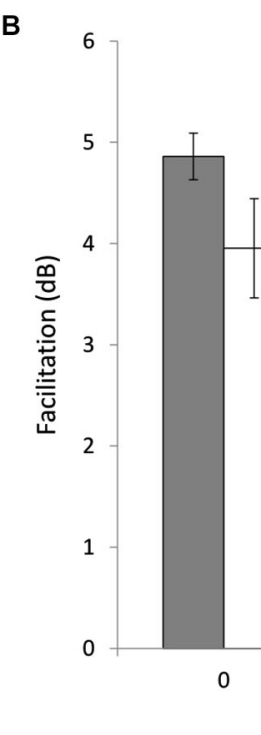

FIGURE 4 | Experiment 1 (collinear facilitation). (A) Baseline contrast thresholds for the two groups. (B) The relationship between flanker orientation offset in degrees and mean facilitation in decibels for NT and ASD subjects.
Error bars indicate SE. Facilitation is reduced for both groups with increasing flanker orientation offset and NT performance was significantly higher across flanker condition.

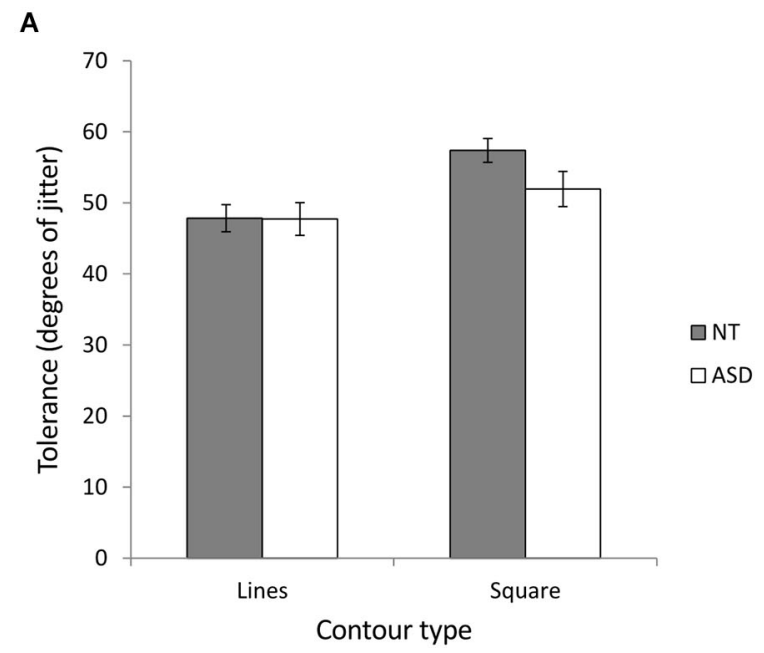

FIGURE 5 | Experiment 2 (contour integration). (A) The relationship between jitter tolerance (the level of jitter at which a participant could successfully detect the target with $75 \%$ accuracy) and the detection of open (lines) and closed (square) figures for NT and ASD subjects. Higher values indicate better detection performance. The NT group show a larger difference in jitter tolerance between lines and squares as indicated by a significant group $\times$
B

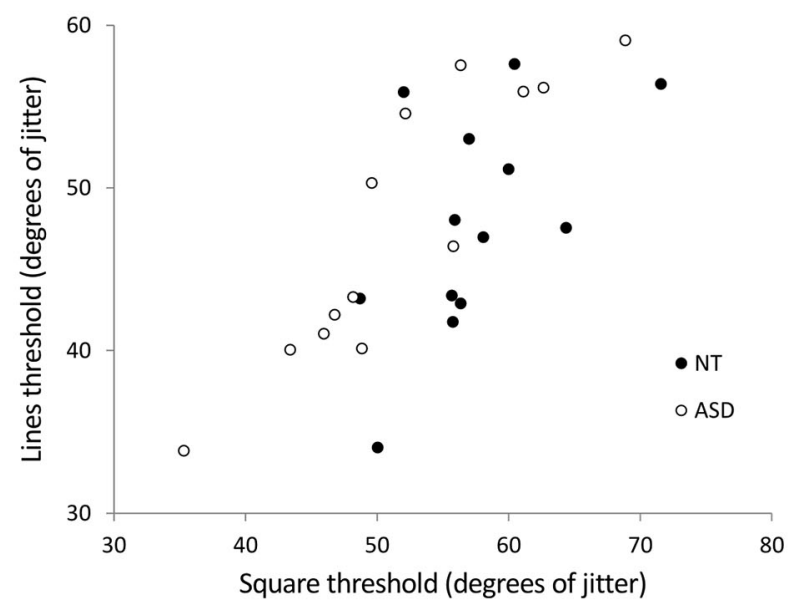

contour-type interaction. Error bars indicate SE. (B) Scatterplot showing the correlation between contour integration performance (tolerance to jitter, higher is better) for lines and square stimuli for the NT and ASD groups. The correlation was stronger for the ASD group $(r=0.90)$ compared to the NT group $(r=0.56)$ which may suggest similar mechanisms underlying the processing of open (lines) and closed (square) stimuli in the case of the ASD group. could tolerate significantly more jitter with the square stimulus $\left(M=57.38^{\circ}, S D=6.01\right)$ than the line stimulus $\left(M=47.83^{\circ}\right.$, $\left.S D=6.86 ; t_{(12)}=5.64, p<0.001\right)$. Although ASD participants could also tolerate significantly more jitter with the square stimulus $\left(M=51.93^{\circ}, S D=8.95\right)$ than the line stimulus $(M=$ $\left.47.73^{\circ}, S D=8.28 ; t_{(12)}=3.80, p=0.003\right)$, this improvement was significantly less than that shown by the NT group, a finding which may indicate reduced visual integration in autism. Simple main effects comparison indicated that the ASD and NT group did not significantly differ for the lines $\left(t_{(24)}=0.036, p=0.972\right)$ or square $\left(t_{(24)}=1.82, p=0.081\right)$ stimuli. Pearson correlation coefficients were used to examine any relationship between the processing of the two stimuli. Both the NT $(r=0.56, p=0.048)$ and the ASD $(r=0.90$, 


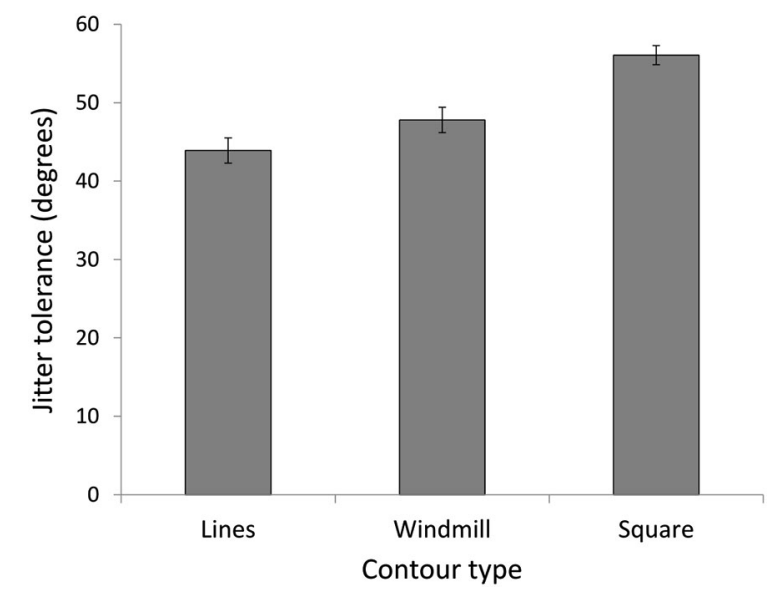

FIGURE 6 | Experiment 3. Jitter tolerance in degrees at which NT participants could detect the stimuli with $75 \%$ accuracy. Results confirm predictions of easier detection for a closed contour (square), even when the open contour (windmill) had the same number of elements as the closed contour (square).

$p<0.001)$ groups showed significant correlations between the integration of the lines and the integration of the square (Figure 5B). In order to directly compare these correlation coefficients between the two groups, the correlation coefficients were converted to z-scores using Fisher's r-to-z transformation (Fisher, 1915). Comparison of these $\mathrm{z}$ scores (Cohen and Cohen, 1983) revealed that the group difference between these r-values just missed significance $(Z=1.88 ; p=0.06)$.

In experiment 3 , we investigated $\mathrm{CI}$ for three stimulus conditions (lines, windmill, square) in a new group of NT participants, to assess whether the increased detection for the NT group with the square stimulus in Expt. 2 was was the result of global enhancement or simply more stimulus information. A repeated measures ANOVA showed a main effect for stimulus $\left(F_{(3,42)}=49.05, p=0.000, \eta_{P}^{2}=0.78\right)$ (Figure 6). As predicted, the windmill $(M=47.80, S D=6.28)$, was easier to identify than the lines $\left(M=43.90, S D=6.18 ; t_{(14)}=3.32, p=0.015\right)$ but harder to identify than the square $\left(M=56.07, S D=4.69 ; t_{(14)}=5.83\right.$, $p<0.0001)$.

\section{Discussion}

We investigated visuospatial integration in participants with ASD by examining collinear facilitation at four flanker orientation offsets, and contour integration for open and closed contours. In support of altered integration, results indicated that collinear facilitation was reduced for the ASD group, and that the detection advantage for closed contours was smaller for the ASD group.

For the collinear facilitation experiment, both our groups demonstrated the usual pattern of performance, showing decreasing facilitation with increasing flanker orientation offset. However the ASD group demonstrated reduced collinear facilitation compared to the control group, which was significant for the $15^{\circ}$ offset. As contrast sensitivities for the target in the baseline (no flanker) condition were similar for both groups, reduced collinear facilitation in the ASD group cannot be attributed to decreased visual acuity. The baseline condition is consistent with previous findings suggesting no difference in visual acuity (Falkmer et al., 2011; Albrecht et al., 2014) or contrast sensitivity (Koh et al., 2010) for equivalent spatial frequencies between ASD and NT groups.

Our results showing reduced or equivalent collinear facilitation contradict those of Kéita et al. (2011) who reported increased collinear facilitation at $3 \lambda$ for adults with ASD relative to controls. As ASD is a heterogeneous condition it is possible that the two studies are testing different subgroups within the population. Indeed, our participant group is composed of individuals diagnosed with Asperger's and has a much higher IQ compared to those in the study by Kéïta et al. (120 vs. 100) which excluded Asperger's. However, there are also several differences between our study and that of Kéita et al. (2011). For example, Kéïta et al. used a longer stimulus presentation time $(500 \mathrm{~ms}$ compared to $100 \mathrm{~ms}$ ) and added grayscale noise to the target and flankers, a manipulation that has been shown to reduce facilitation (Huang and Hess, 2007). The addition of grayscale noise may account for the similar levels of facilitation found by Kéita et al. at both $6 \lambda$ and $3 \lambda$ flanker conditions for controls, a result that contradicts a substantial body of previous collinear facilitation studies indicating greater facilitation at $3 \lambda$ (Polat and Sagi, 1993, 1994, 2006, 2007; Polat and Norcia, 1996; Adini et al., 1997; Polat, 1999; Huang et al., 2006; Huang and Hess, 2007; Sterkin et al., 2009). In contrast, our NT results are consistent with levels observed at $3 \lambda$ separations in these previous studies. The current findings raise the possibility that the ASD group in Kéita et al. (2011) were less affected by the noise than controls, but future work comparing flanker separations with and without noise is required.

If collinear facilitation is assumed to rely on horizontal connectivity in V1 for the integration of the flanker signals at the target site (Polat and Sagi, 2007), then reduced facilitation could be the result of fewer, longer or slower connections between flanking and target neurons. Morphological studies however, suggest a bias towards shorter V1 connections in autism (Casanova et al., 2006). One possible explanation for the reduced facilitation shown by participants with ASD could lie with the temporal integration of the visual stimuli. Although the target and flankers appear onscreen simultaneously, there are two sources of delay that impact integration of the flanker and target signals in V1. Firstly, because of temporal coding of contrast (Reich et al., 2001) and the low contrast of the target stimuli, target neurons respond later than flanker neurons to the visual input. Secondly, signals from the flanker sites take time to reach the target site because of the relatively slow propagation rate of signals along horizontal connections (Bringuier et al., 1999; Cass and Spehar, 2005). These delays determine the temporal window, or overlap, during which integration of signals from the flankers and target can occur. Differences in these delays between the ASD and NT groups may therefore contribute to the decrease in facilitation shown by the ASD group. This idea complements theories suggesting altered temporal processing in autism (Gepner and Féron, 2009) and evidence for poorer 
performance on temporal perception tasks (de Boer-Schellekens et al., 2013).

Altered top-down processing in autism (Frith, 2003) may also be a factor in reduced collinear facilitation. Huang and Hess (2008) showed that collinear facilitation occurred when the target was presented before the flankers, a result that is difficult to reconcile with the horizontal connectivity account given above (Polat and Sagi, 2006). They propose an additional facilitating mechanism based on rapid orientation-specific feedback from V2 (Girard et al., 2001). If this feedback is attenuated in ASD, then facilitation may be impaired. Another top-down process that can modulate collinear facilitation is attention; facilitation depends on whether or not the flankers are attended (Freeman et al., 2003). Therefore, reduced attentional feedback may also influence lateral interactions in V1 (Gilbert et al., 2000).

Turning to the contour integration experiment, both groups showed a significant improvement viewing the square stimulus, a finding consistent with previous work demonstrating easier detection of closed compared to open contours (Kovács and Julesz, 1993; Mathes and Fahle, 2007). However, the significant interaction revealed a greater effect of closure for the NT group, indicating that altered contour integration in ASD adults is apparent for the detection of simple shapes. Experiment 3 was designed to rule out the possibility that increased visual information (more Gabors) in the square compared to the lines may have driven the enhancement of the square and showed that while the windmill stimulus led to better contour detection than the lines, contour integration for the closed square stimulus was still better than the windmill. In line with previous results that have equated Gabor number between closed and open stimuli (Mathes and Fahle, 2007), these findings suggest that the number of Gabors do play a role, but that NT participants are also sensitive to the closed nature of the square stimulus. It remains possible that the presence of corners in the square compared to windmill may contribute to enhanced perception of the square due to "good continuation" rather than closure. Against this argument is evidence for a closure mechanism when factors such as good continuation have been controlled for (Gerhardstein et al., 2012).

There is some debate as to the exact mechanism behind the improvement in contour detection seen with closure. Low-level explanations suggest that this improvement results from the properties of the horizontal connections within V1 (Pettet et al., 1998; Yen and Finkel, 1998; Tversky et al., 2004). For example, facilitation between similarly orientated elements may be able to propagate multiple times around V1 cells representing the closed contour, enhancing contour integration, termed "reverberation" (Pettet et al., 1998; Yen and Finkel, 1998). However, in support of a distinct closure detection mechanism, Gerhardstein et al. (2012) showed that these low-level accounts cannot fully account for closure, and suggested the need for a separate global or semi-global mechanism, speculating on the role of V4 as a global shape processor (Pasupathy and Connor, 2002). Physiological evidence also points towards a top-down influence in the perception of closed contours. Using fMRI, Murray et al. (2002) studied brain activity whilst human subjects viewed simple line drawings of random lines, $2 \mathrm{D}$ shapes and $3 \mathrm{D}$ shapes. This progression from open to closed stimuli was positively correlated with activity in the LOC, and negatively correlated with activity in V1. The authors suggest that grouping-processes at higher levels generate feedback which reduces activity in lower areas. Furthermore, Gilad et al. (2013) trained macaques to perform a closed-contour integration task and monitored their V1 activity using voltage-sensitive dye imaging. They observed the initial brightening of $\mathrm{V} 1$ regions representing the contour Gabors, followed by dimming of $\mathrm{V} 1$ regions representing the background Gabors. Both of these events had the effect of increasing contour saliency. Gilad et al. suggest that the initial brightening is mediated by horizontal as well as feedback connections, whereas the later background suppression may be the result of changes in top-down feedback to V1.

Therefore, it is possible that the reduced effect of closure in the ASD group results from altered top down feedback, a suggestion that receives indirect support from two aspects of our findings. Firstly, the "lines" compared to "square" improvement shown by the ASD group is similar to the "lines" compared to "windmill" improvement demonstrated by the NT follow-up group. This comparison could be interpreted as the ASD group benefiting from the extra contour information in the square, but not receiving a top-down "boost" to further augment contour integration to NT levels. Secondly, the stronger correlation between the lines and the square results for the ASD group compared to NT group (Figure 5) was reaching significance, suggesting that similar processing mechanisms may be at work for both stimuli in the ASD group, but that different mechanisms (i.e., feedback) may also be operating in the NT group, when processing closed stimuli. A reduction in modulatory feedback is consistent with a predictive coding model of visual perception (Rao and Ballard, 1999), in which higher brain areas make predictions about input from lower brain areas, and then feedback these predictions to lower areas, where the predicted sensory information is compared to the actual sensory input. Altered predictive coding, such as the generation of less precise predictions has recently been forwarded as an explanation for the perceptual differences found in autism including the decrease in global processing (van Boxtel and Lu, 2013).

While our comparison between lines and squares has provided novel evidence for altered contour integration in $\mathrm{ASD},{ }^{2}$ no significant differences were found when directly comparing the participant groups for either the lines or the square stimuli, supporting previous psychophysical research investigating contour integration for simple shapes in autism (Del Viva et al., 2006; Kemner et al., 2007). One limitation of our work is that it lacked power to obtain significant group differences: In order to achieve a power of 0.8 for comparison

\footnotetext{
${ }^{2} \mathrm{An}$ interaction refers to the comparison of within group differences NOT between group simple main effects (Nieuwenhuis et al., 2011). Our interaction is sufficient evidence to show that the groups differ and simple main effects analysis between groups is not necessary for interpretation of the interaction. We added the simple main effects comparison only to compare with previous behavioural contour integration experiments. Therefore, low power for simple main effects analysis does not detract from our principle finding of a significant interaction (with large effect size) between group and stimulus.
} 
of the square stimulus, 24 participants in each group would be required. Consequently, our results highlight that a lack of power may have also contributed to previous negative findings using between groups analysis, but that the interaction between open and closed contours reveals a much stronger effect. Evidence of group differences with larger group sizes has recently been reported (Evers et al., 2014), although as this used a recognition task it also raises the possibility that group differences in contour integration may become apparent with more complex tasks.

In summary, we employed two psychophysical paradigms, collinear facilitation and contour integration to investigate visual integration in adults with ASD. The ASD group showed reduced

\section{References}

Achtman, R. L., Hess, R. F., and Wang, Y. Z. (2003). Sensitivity for global shape detection. J. Vis. 3, 616-624. doi: 10.1167/3.10.4

Adini, Y., Sagi, D., and Tsodyks, M. (1997). Excitatory-inhibitory network in the visual cortex: psychophysical evidence. Proc. Natl. Acad. Sci. U S A 94, 10426-10431. doi: 10.1073/pnas.94.19.10426

Albrecht, M. A., Stuart, G. W., Falkmer, M., Ordqvist, A., Leung, D., and Foster, J. K. (2014). Brief report: visual acuity in children with autism spectrum disorders. J. Autism Dev. Disord. 44, 2369-2374. doi: 10.1007/s10803-0142086-x

Altmann, C. F., Bülthoff, H. H., and Kourtzi, Z. (2003). Perceptual organization of local elements into global shapes in the human visual cortex. Curr. Biol. 13, 342-349. doi: 10.1016/s0960-9822(03)00052-6

American Psychiatric Association. (1994). Diagnostic and Statistical Manual of Mental Disorders: DSM-IV. Washington DC: American Psychiatric Pub Inc.

American Psychiatric Association. (2013). DSM 5. Washington DC: American Psychiatric Association.

Angelucci, A., Levitt, J. B., Walton, E. J., Hupe, J. M., Bullier, J., and Lund, J. S. (2002). Circuits for local and global signal integration in primary visual cortex. J. Neurosci. 22, 8633-8646.

Blake, R., Turner, L. M., Smoski, M. J., Pozdol, S. L., and Stone, W. L. (2003). Visual recognition of biological motion is impaired in children with autism. Psychol. Sci. 14, 151-157. doi: 10.1111/1467-9280.01434

Blakemore, C., Carpenter, R. H., and Georgeson, M. A. (1970). Lateral inhibition between orientation detectors in the human visual system. Nature 228, 37-39. doi: $10.1038 / 228037 \mathrm{a} 0$

Bogdashina, O. (2003). Sensory Perceptual Issues in Autism and asperger Syndrome: Different Sensory Experiences-Different Perceptual Worlds. London: Jessica Kingsley Publishers.

Brainard, D. H. (1997). The psychophysics toolbox. Spat. Vis. 10, 433-436. doi: 10. 1163/156856897x00357

Bringuier, V., Chavane, F., Glaeser, L., and Frégnac, Y. (1999). Horizontal propagation of visual activity in the synaptic integration field of area 17 neurons. Science 283, 695-699. doi: 10.1126/science.283.5402.695

Casanova, M. F., van Kooten, I. A., Switala, A. E., van Engeland, H., Heinsen, H., Steinbusch, H. W., et al. (2006). Minicolumnar abnormalities in autism. Acta Neuropathol. 112, 287-303. doi: 10.1007/s00401-006-0085-5

Cass, J. R., and Spehar, B. (2005). Dynamics of collinear contrast facilitation are consistent with long-range horizontal striate transmission. Vision Res. 45, 2728-2739. doi: 10.1016/j.visres.2005.03.010

Cohen, J., and Cohen, P. (1983). Applied Multiple Regression/Correlation Analysis for the Behavioral Sciences. Hillsdale, NJ: Erlbaum.

Dakin, S. C., and Baruch, N. J. (2009). Context influences contour integration. J. Vis. 9:13. doi: 10.1167/9.2.13

Dakin, S., and Frith, U. (2005). Vagaries of visual perception in autism. Neuron 48, 497-507. doi: 10.1016/j.neuron.2005.10.018

de Boer-Schellekens, L., Eussen, M., and Vroomen, J. (2013). Diminished sensitivity of audiovisual temporal order in autism spectrum disorder. Front. Integr. Neurosci. 7:8. doi: 10.3389/fnint.2013.00008 collinear facilitation and less improvement in contour detection between open and closed contours compared to the NT group. These results, together with previous studies using different psychophysical approaches (Vandenbroucke et al., 2009) indicate weaker visuospatial integration in adults with ASD, which may underlie perceptual superiority in tasks that benefit from ignoring contextual information.

\section{Author Contributions}

All authors contributed towards the design, interpretation, writing and revision of the manuscript. SJ performed data collection, programming of experiments and analysis.

Del Viva, M. M., Igliozzi, R., Tancredi, R., and Brizzolara, D. (2006). Spatial and motion integration in children with autism. Vision Res. 46, 1242-1252. doi: 10. 1016/j.visres.2005.10.018

Evers, K., Panis, S., Torfs, K., Steyaert, J., Noens, I., and Wagemans, J. (2014). Disturbed interplay between mid- and high-level vision in ASD? Evidence from a contour identification task with everyday objects. J. Autism Dev. Disord. 44, 801-815. doi: 10.1007/s10803-013-1931-7

Falkmer, M., Stuart, G. W., Danielsson, H., Bram, S., Lönebrink, M., and Falkmer, T. (2011). Visual acuity in adults with Asperger's syndrome: no evidence for “eagle-eyed” vision. Biol. Psychiatry 70, 812-816. doi: 10.1016/j.biopsych.2011. 07.025

Faul, F., Erdfelder, E., Lang, A. G., and Buchner, A. (2007). G* power 3: a flexible statistical power analysis program for the social, behavioral and biomedical sciences. Behav. Res. Methods 39, 175-191. doi: 10.3758/bf03193146

Field, D. J., Hayes, A., and Hess, R. F. (1993). Contour integration by the human visual system: evidence for a local "association field". Vision Res. 33, 173-193. doi: 10.1016/0042-6989(93)90156-q

Fisher, R. A. (1915). Frequency distribution of the values of the correlation coefficient in samples of an indefinitely large population. Biometrika 10, 507521. doi: $10.2307 / 2331838$

Fitzpatrick, D. (1996). The functional organization of local circuits in visual cortex: insights from the study of tree shrew striate cortex. Cereb. Cortex 6, 329-341. doi: $10.1093 /$ cercor/6.3.329

Freeman, E., Driver, J., Sagi, D., and Zhaoping, L. (2003). Top-down modulation of lateral interactions in early vision: does attention affect integration of the whole or just perception of the parts? Curr. Biol. 13, 985-989. doi: 10.1016/s09609822(03)00333-6

Freeman, E., Sagi, D., and Driver, J. (2004). Configuration-specific attentional modulation of flanker-target lateral interactions. Perception 33, 181-194. doi: $10.1068 / \mathrm{p} 3481$

Frith, U. (2003). Autism: Explaining the Enigma. London: Blackwell Publishing.

Gepner, B., and Féron, F. (2009). Autism: a world changing too fast for a mis-wired brain? Neurosci. Biobehav. Rev. 33, 1227-1242. doi: 10.1016/j.neubiorev.2009. 06.006

Gerhardstein, P., Tse, J., Dickerson, K., Hipp, D., and Moser, A. (2012). The human visual system uses a global closure mechanism. Vision Res. 71, 18-27. doi: 10. 1016/j.visres.2012.08.011

Gheorghiu, E., Kingdom, F. A., and Petkov, N. (2014). Contextual modulation as de-texturizer. Vision Res. 104, 12-23. doi: 10.1016/j.visres.2014.08.013

Gilad, A., Meirovithz, E., and Slovin, H. (2013). Population responses to contour integration: early encoding of discrete elements and late perceptual grouping. Neuron 78, 389-402. doi: 10.1016/j.neuron.2013.02.013

Gilbert, C., Ito, M., Kapadia, M., and Westheimer, G. (2000). Interactions between attention, context and learning in primary visual cortex. Vision Res. 40, 1217-1226. doi: 10.1016/s0042-6989(99)00234-5

Gilbert, C. D., and Wiesel, T. N. (1983). Clustered intrinsic connections in cat visual cortex. J. Neurosci. 3, 1116-1133.

Gilbert, C. D., and Wiesel, T. N. (1989). Columnar specificity of intrinsic horizontal and corticocortical connections in cat visual cortex. J. Neurosci. 9 , 2432-2442. 
Girard, P., Hupé, J., and Bullier, J. (2001). Feedforward and feedback connections between areas V1 and V2 of the monkey have similar rapid conduction velocities. J. Neurophysiol. 85, 1328-1331.

Happé, F. G. E. (1996). Studying weak central coherence at low levels: children with autism do not succumb to visual illusions. A research note. J. Child Psychol. Psychiatry 37, 873-877. doi: 10.1111/j.1469-7610.1996.tb01483.x

Happé, F., and Frith, U. (2006). The weak coherence account: detail-focused cognitive style in autism spectrum disorders. J. Autism Dev. Disord. 36, 5-25. doi: 10.1007/s10803-005-0039-0

Hartline, H. K. (1949). Inhibition of activity of visual receptors by illuminating nearby retinal areas in the Limulus eye. Fed. Proc. 8, 69.

Huang, P. C., and Hess, R. F. (2007). Collinear facilitation: effect of additive and multiplicative external noise. Vision Res. 47, 3108-3119. doi: 10.1016/j.visres. 2007.08.007

Huang, P. C., and Hess, R. F. (2008). The dynamics of collinear facilitation: fast but sustained. Vision Res. 48, 2715-2722. doi: 10.1016/j.visres.2008.09.013

Huang, P. C., Hess, R. F., and Dakin, S. C. (2006). Flank facilitation and contour integration: different sites. Vision Res. 46, 3699-3706. doi: 10.1016/j.visres. 2006.04.025

Hupé, J., James, A. C., Payne, B. R., Lomber, S. G., Girard, P., and Bullier, J. (1998). Cortical feedback improves discrimination between figure and background by V1, V2 and V3 neurons. Nature 394, 784-787. doi: 10.1038/29537

Kapadia, M. K., Ito, M., Gilbert, C. D., and Westheimer, G. (1995). Improvement in visual sensitivity by changes in local context: parallel studies in human observers and in V1 of alert monkeys. Neuron 15, 843-856. doi: 10.1016/08966273(95)90175-2

Kapadia, M. K., Westheimer, G., and Gilbert, C. D. (2000). Spatial distribution of contextual interactions in primary visual cortex and in visual perception. $J$. Neurophysiol. 84, 2048-2062.

Kéïta, L., Mottron, L., Dawson, M., and Bertone, A. (2011). Atypical lateral connectivity: a neural basis for altered visuospatial processing in autism. Biol. Psychiatry 70, 806-811. doi: 10.1016/j.biopsych.2011.07.031

Kemner, C., Lamme, V. A., Kovacs, I., and van Engeland, H. (2007). Integrity of lateral and feedbackward connections in visual processing in children with pervasive developmental disorder. Neuropsychologia 45, 1293-1298. doi: 10. 1016/j.neuropsychologia.2006.09.016

Kingdom, F., and Prins, N. (2009). Psychophysics: A Practical Introduction. London: Academic Press.

Koh, H. C., Milne, E., and Dobkins, K. (2010). Spatial contrast sensitivity in adolescents with autism spectrum disorders. J. Autism Dev. Disord. 40, 978-987. doi: 10.1007/s10803-010-0953-7

Kontsevich, L. L., and Tyler, C. W. (1999). Bayesian adaptive estimation of psychometric slope and threshold. Vision Res. 39, 2729-2737. doi: 10. 1016/s0042-6989(98)00285-5

Kovács, I., and Julesz, B. (1993). A closed curve is much more than an incomplete one: effect of closure in figure-ground segmentation. Proc. Natl. Acad. Sci. US A 90, 7495-7497. doi: 10.1073/pnas.90.16.7495

Li, X., Lu, Z. L., Xu, P., Jin, J., and Zhou, Y. (2003). Generating high graylevel resolution monochrome displays with conventional computer graphics cards and color monitors. J. Neurosci. Methods 130, 9-18. doi: 10.1016/s01650270(03)00174-2

Livingstone, M. S., and Hubel, D. H. (1984). Specificity of intrinsic connections in primate primary visual cortex. J. Neurosci. 4, 2830-2835.

Loffler, G. (2008). Perception of contours and shapes: low and intermediate stage mechanisms. Vision Res. 48, 2106-2127. doi: 10.1016/j.visres.2008.03.006

Lord, C., Risi, S., Lambrecht, L., Cook, E. H. Jr., Leventhal, B. L., DiLavore, P. C., et al. (2000). The autism diagnostic observation schedule - generic: a standard measure of social and communication deficits associated with the spectrum of autism. J. Autism Dev. Disord. 30, 205-223. doi: 10.1023/A:10055924 01947

Mathes, B., and Fahle, M. (2007). Closure facilitates contour integration. Vision Res. 47, 818-827. doi: 10.1016/j.visres.2006.11.014

Mijović, B., De Vos, M., Vanderperren, K., Machilsen, B., Sunaert, S., Van Huffel, S., et al. (2014). The dynamics of contour integration: a simultaneous EEG-fMRI study. Neuroimage 88, 10-21. doi: 10.1016/j.neuroimage.2013. 11.032

Murray, S. O., Kersten, D., Olshausen, B. A., Schrater, P., and Woods, D. L. (2002). Shape perception reduces activity in human primary visual cortex. Proc. Natl. Acad. Sci. U S A 99, 15164-15169. doi: 10.1073/pnas.192579399
Nieuwenhuis, S., Forstmann, B. U., and Wagenmakers, E.-J. (2011). Erroneous analyses of interactions in neuroscience: a problem of significance. Nat. Neurosci. 14, 1105-1107. doi: 10.1038/nn.2886

Nurminen, L., and Angelucci, A. (2014). Multiple components of surround modulation in primary visual cortex: multiple neural circuits with multiple functions? Vision Res. 104, 47-56. doi: 10.1016/j.visres.2014. 08.018

Pasupathy, A., and Connor, C. E. (2002). Population coding of shape in area V4. Nat. Neurosci. 5, 1332-1338. doi: 10.1038/972

Pei, F., Baldassi, S., Procida, G., Igliozzi, R., Tancredi, R., Muratori, F., et al. (2009). Neural correlates of texture and contour integration in children with autism spectrum disorders. Vision Res. 49, 2140-2150. doi: 10.1016/j.visres.2009. 06.006

Pelli, D. G. (1997). The VideoToolBox software for visual psychophysics: transforming numbers into movies. Spat. Vis. 10, 437-442. doi: 10 $1163 / 156856897 \times 00366$

Pettet, M. W., McKee, S. P., and Grzywacz, N. M. (1998). Constraints on long range interactions mediating contour detection. Vision Res. 38, 865-879. doi: 10 1016/s0042-6989(97)00238-1

Polat, U. (1999). Functional architecture of long-range perceptual interactions. Spat. Vis. 12, 143-162. doi: 10.1163/156856899x00094

Polat, U., and Norcia, A. M. (1996). Neurophysiological evidence for contrast dependent long-range facilitation and suppression in the human visual cortex. Vision Res. 36, 2099-2109. doi: 10.1016/0042-6989(95) 00281-2

Polat, U., and Sagi, D. (1993). Lateral interactions between spatial channels: suppression and facilitation revealed by lateral masking experiments. Vision Res. 33, 993-999. doi: 10.1016/0042-6989(93)90081-7

Polat, U., and Sagi, D. (1994). The architecture of perceptual spatial interactions. Vision Res. 34, 73-78. doi: 10.1016/0042-6989(94)90258-5

Polat, U., and Sagi, D. (2006). Temporal asymmetry of collinear lateral interactions. Vision Res. 46, 953-960. doi: 10.1016/j.visres.2005. 09.031

Polat, U., and Sagi, D. (2007). The relationship between the subjective and objective aspects of visual filling-in. Vision Res. 47, 2473-2481. doi: 10.1016/j. visres.2007.06.007

Rao, R. P. N., and Ballard, D. H. (1999). Predictive coding in the visual cortex: a functional interpretation of some extra-classical receptive-field effects. Nat. Neurosci. 2, 79-87. doi: 10.1038/4580

Reich, D. S., Mechler, F., and Victor, J. D. (2001). Temporal coding of contrast in primary visual cortex: when, what and why. J. Neurophysiol. 85, 1039-1050.

Rockland, K. S., and Lund, J. S. (1982). Widespread periodic intrinsic connections in the tree shrew visual cortex. Science 215, 1532-1534. doi: 10.1126/science. 7063863

San Agustin, J., Skovsgaard, H., Mollenbach, E., Barret, M., Tall, M., Hansen, D. W., et al (2010). "Evaluation of a low-cost open-source gaze tracker," in Proceedings of the 2010 Symposium on Eye-Tracking Research and Applications (New York, NY), 77-80.

Schmid, A. M., and Victor, J. D. (2014). Possible functions of contextual modulations and receptive field nonlinearities: pop-out and texture segmentation. Vision Res. 104, 57-67. doi: 10.1016/j.visres.2014.07.002

Schumacher, J. F., Quinn, C. F., and Olman, C. A. (2011). An exploration of the spatial scale over which orientation-dependent surround effects affect contour detection. J. Vis. 11:12. doi: 10.1167/11.8.12

Shah, A., and Frith, U. (1983). An islet of ability in autistic children: a research note. J. Child Psychol. Psychiatry 24, 613-620. doi: 10.1111/j.1469-7610.1983. tb00137.x

Shmuel, A., Korman, M., Sterkin, A., Harel, M., Ullman, S., Malach, R., et al. (2005). Retinotopic axis specificity and selective clustering of feedback projections from V2 to V1 in the owl monkey. J. Neurosci. 25, 2117-2131. doi: 10.1523/jneurosci.4137-04.2005

Shpaner, M., Molholm, S., Forde, E., and Foxe, J. J. (2013). Disambiguating the roles of area V1 and the lateral occipital complex (LOC) in contour integration. Neuroimage 69, 146-156. doi: 10.1016/j.neuroimage.2012. 11.023

Shushruth, S., Nurminen, L., Bijanzadeh, M., Ichida, J. M., Vanni, S., and Angelucci, A. (2013). Different orientation tuning of near-and far-surround suppression in macaque primary visual cortex mirrors their tuning in 
human perception. J. Neurosci. 33, 106-119. doi: 10.1523/jneurosci.2518-12. 2013

Simmons, D. R., Robertson, A. E., McKay, L. S., Toal, E., McAleer, P., and Pollick, F. E. (2009). Vision in autism spectrum disorders. Vision Res. 49, 2705-2739. doi: 10.1016/j.visres.2009.08.005

Sterkin, A., Yehezkel, O., Bonneh, Y. S., Norcia, A., and Polat, U. (2009). Backward masking suppresses collinear facilitation in the visual cortex. Vision Res. 49, 1784-1794. doi: 10.1016/j.visres.2009.04.013

Stettler, D. D., Das, A., Bennett, J., and Gilbert, C. D. (2002). Lateral connectivity and contextual interactions in macaque primary visual cortex. Neuron 36, 739-750. doi: 10.1016/s0896-6273(02)01029-2

Tversky, T., Geisler, W. S., and Perry, J. S. (2004). Contour grouping: closure effects are explained by good continuation and proximity. Vision Res. 44, 2769-2777. doi: 10.1016/j.visres.2004.06.011

van Boxtel, J. J., and Lu, H. (2013). A predictive coding perspective on autism spectrum disorders. Front. Psychol. 4:19. doi: 10.3389/fpsyg.2013. 00019

Vandenbroucke, M. W. G., Scholte, H. S., van Engeland, H., Lamme, V. A., and Kemner, C. (2009). A new approach to the study of detail perception in autism spectrum disorder (ASD): investigating visual feedforward, horizontal and feedback processing. Vision Res. 49, 1006-1016. doi: 10.1016/j.visres.2007. 12.017

Van der Hallen, R., Evers, K., Brewaeys, K., Van den Noortgate, W., and Wagemans, J. (2014). Global processing takes time: a meta-analysis on local-global visual processing in ASD. Psychol. Bull. doi: 10.1037/bul0000004. [Epub ahead of print].

Wechsler, D. (1999). Wechsler Abbreviated Scale of Intelligence. San Antonio, TX: The Psychological Corporation.

Wichmann, F. A., and Hill, N. J. (2001). The psychometric function: I. Fitting, sampling and goodness of fit. Percept. Psychophys. 63, 1293-1313. doi: 10 3758/bf03194544

World Health Organization. (1993). The ICD-10 Classification of Mental and Behavioural Disorders: Diagnostic Criteria for Research. Geneva: World Health Organization.

Yen, S. C., and Finkel, L. H. (1998). Extraction of perceptually salient contours by striate cortical networks. Vision Res. 38, 719-741. doi: 10.1016/s00426989(97)00197-1

Conflict of Interest Statement: The authors declare that the research was conducted in the absence of any commercial or financial relationships that could be construed as a potential conflict of interest.

Copyright (c) 2015 Jachim, Warrens, McLoughlin and Gowen. This is an open-access article distributed under the terms of the Creative Commons Attribution License (CC $B Y)$. The use, distribution and reproduction in other forums is permitted, provided the original author(s) or licensor are credited and that the original publication in this journal is cited, in accordance with accepted academic practice. No use, distribution or reproduction is permitted which does not comply with these terms. 